\title{
Venezuela no es más una democracia
}

\author{
Venezuela não é mais uma democracia \\ Venezuela is Not a Democracy Anymore
}

Lucas Souto Ribeiro*

\begin{abstract}
Palabras clave:
Venezuela, democracia, socialismo, chavismo.

Resumén: Venezuela viene sufriendo un proceso de deterioro institucional en las últimas décadas con la ascensión del modelo del Socialismo del Siglo XXI implementado por Hugo Chávez y seguido por Nicolas Maduro. Este artículo busca comprender si la actual situación de Venezuela es todavía una democracia o no. El estudio se basa en diversos índices internacionales que ayudan a delimitar la situación, y en el referencial conceptual de democracia de Dahl y Levitsky.
\end{abstract}

\section{Palavras-chave:}

Venezuela, democracia, socialismo, chavismo.

\begin{tabular}{l}
\hline Keywords: \\
Venezuela, \\
democracy, \\
socialism, \\
chavism \\
\hline
\end{tabular}

Resumo: Nas últimas décadas, a Venezuela tem sofrido um processo de deteriorização institucional com a ascensão do modelo de Socialismo do Século XXI, implementado por Hugo Chávez e seguido por Nicolas Maduro. Este artigo busca compreender se a Venezuela ainda pode ser considerada uma democracia. O estudo é baseado em diversos índices internacionais que ajudam a delimitar a situação, bem como no referencial conceitual de Dahl e Levitsky.

Abstract: In the last decades, Venezuela has undergone a process of institutional deterioration with the rise of Socialism of the 21st Century, implemented by Hugo Chavez and followed by Nicolas Maduro. This article seeks to understand if Venezuela can still be considered a democracy or not. The study is based on several international indexes that help to define the situation, as well as on the conceptual framework presented by Dahl and Levitsky.

DOI https://doi.org/10.30800/mises.2019.v7.1089

* Graduado en Relaciones Internacionales por la Universidad Unijorge en Brasil, y Magister en Política y Relaciones Internacionales por la Universidad Sergio Arboleda en Colombia. E-mail: falecomlucasribeiro@gmail.com 


\section{Introducción}

La situación en Venezuela sigue empeorando y es un importante debate saber, actualmente, si el régimen es democrático, híbrido o autoritario. En este ensayo buscaremos presentar informaciones de diversos institutos que comparan índices de democracia, estado de derecho, libertades civiles y económicas, y derechos humanos. Todo esto basándose en los marcos teóricos de las maneras de evaluar una democracia como podemos observar en Steven Levistksy, por ejemplo.

Para empezar, presentamos la definición que Levitsky hace de «competitive authoritarianism» sobre lo que un gobierno tiene, algunas estructuras formales de una democracia, aun violando esas mismas instituciones:

This article examines one particular type of "hybrid" regime: competitive authoritarianism. In competitive authoritarian regimes, formal democratic institutions are widely viewed as the principal means of obtaining and exercising political authority. Incumbents violate those rules so often and to such an extent, however, that the regime fails to meet conventional minimum standards for democracy. (LEVITSKY, 2002, p.2)

Otro politólogo importante para definir lo que es una democracia es Robert Dahl (1989). Los requisitos para la democracia son: libertad de asociación, expresión, de voto; elegibilidad para el servicio público; derecho de los líderes políticos a competir, y a luchar por votos; variedad de fuentes de información; elecciones libres e imparciales; e instituciones que garanticen que la expresión de la preferencia influya en la política del gobierno (DAHL, 1989). Y es a partir de los conceptos de Dahl (1989) que vamos a evaluar la democracia en Venezuela.

Buscando informaciones de diversos rankings podemos decir que es un régimen autoritario, y vamos a presentar la fundamentación para tal afirmación. Según el reporte de ranking de democracia de la revista The Economist, en 2017, Venezuela ya presentaba un régimen autoritario. En el reporte de la revista se habla así de Venezuela:

Venezuela becomes an "authoritarian regime" Latin America's average score declined from 6.33 in 2016 to 6.26 in 2017, although the region remains the most democratic in the developing world. Most countries recorded minor changes in their overall scores in 2017. However, two countries in the region shifted categories. Ecuador improved from a "hybrid regime" to a "flawed democracy". Venezuela, by contrast, moved from a "hybrid regime" to an "authoritarian regime", joining Cuba in that category. The latter change reflects Venezuela's continued slide towards dictatorship. (THE ECONOMIST, 2017)

Los criterios de la metodología del Índice de Democracia de revista The Economist es importante para entender cómo se llega en este ranking. Los elementos son: proceso electoral y pluralismo; libertades civiles; funcionamiento gubernamental; participación política y cultura política:

The Economist Intelligence Unit's index of democracy, on a 0 to 10 scale, is based on the ratings for 60 indicators, grouped into five categories: electoral process and pluralism; civil liberties; the functioning of government; political participation; and political culture. Each category has a rating on a 0 to 10 scale, and the overall Index is the simple average of the five category indexes. 
Otro indicador importante para que se pueda medir la calidad de una democracia es la situación de la prensa libre y de los reporteros. Según Reporteros Sin Fronteras (2018), Venezuela está en la posición 143 de 180 y se presenta como un país en «situación difícil» para los periodistas.

Frasier Institute creó en 1986 un ranking para medir la libertad económica de un país. Aunque la democracia no sea apenas eso, este índice ayuda a tener una idea de la situación de las libertades en un país. Venezuela quedó en la última posición (159/159). El mismo instituto tiene otro índice llamado 'Índice de Libertad Humana', en este ranking se considera las libertades civiles, políticas y económicas, y nuevamente Venezuela queda en la última posición (FRASIER INSTITUTE, 2017, s/p).

Un aspecto decisivo para una democracia saludable es la calidad del sistema judicial y sus instituciones. "World Justice Project" creó un índice que evalúa la calidad del Estado de Derecho ("Rule of Law") de 113 países. Venezuela en los subíndices del "Rule of Law Index" quedó en la posición: 110/113 en gobierno abierto; 105/113 en derechos fundamentales; 110/113 en orden y seguridad; 113/113 en cumplimiento regulatorio; 112/113 en justicia civil, y 113/113 en justicia penal (RULE OF LAW INDEX, 2018).

Además de libertades, prensa libre, y Estado de Derecho, los derechos humanos son un fundamento básico para la calidad de una democracia. A Humans Right Watch es una ONG experta en derechos humanos. Vamos a observar el análisis cualitativo hecho por HRW. Según Humans Right Watch el poder judiciario ya no es independiente desde 2004 y quedó como una rama subordinada al poder ejecutivo:

Judicial Independence: Since former President Chávez and his supporters in the National Assembly conducted a political takeover of the Supreme Court in 2004, the judiciary has ceased to function as an independent brach of government. Members of the Supreme Court have openly rejected the principle of separation of powers, and publicly pledged their commitment to advancing the current administration's political agenda. Since the opposition assumed the majority in the National Assembly in January 2016, the Supreme Court has struck down almost every law it has passed. In March 2017, it took over all legislative powers, and partially backtracked only after strong criticism in Venezuela and abroad. (HUMAN RIGHT WATCH, 2018)

En términos de la división de poderes, Maduro convocó una Asamblea Constituyente en mayo de 2017 para tener un poder legislativo con sus aliados (HUMANS WATCH REPORT, 2018). Además de eso, la persecución y encarcelamiento de opositores políticos es algo presente en la dictadura chavista, y tenemos la prisión de Leopoldo Lopez y de Antonio Ledesma, conforme dijo el reporte de Humans Right Watch:

Opposition leader Leopoldo López is serving a 13-year sentence for allegedly inciting violence during a demonstration in Caracas in February 2014, despite the lack of any credible evidence against him. After three-and-a-half years in prison, López was moved to house arrest in July 2017, but was again detained in the middle of the night weeks later after he publicly criticized the government. That same night, intelligence agents detained Antonio Ledezma, a former opposition mayor who has been under house arrest since 2015 and had published a critical video while under house arrest. (HUMAN RIGHT WATCH, 2018) 
Hay también una acción de los «colectivos» que son grupos paramilitares que son usados para atacar a la oposición. Y más de 100 personas fueron muertas según la Fiscalía General de Venezuela, y más de 5 mil personas fueron detenidas por razones políticas (HUMAN RIGHT WATCH, 2018).

En términos de libertad y derechos humanos hay graves violaciones por parte de la dictadura de Maduro. Human Right Watch revela que ha sido creada una «ley de crimines de odio» donde se habla de manera vaga de «fascismo, odio e intolerancia» de manera que se pueda encarcelar a opositores. Según el mismo reporte Organizaciones de Derechos Humanos internacionales puede enjuiciarse por «crímenes de traición» y «contra la estabilidad democrática» en Venezuela. (HUMAN RIGHT WATCH, 2018). En el escenario internacional el Mercosur suspendió a Venezuela en 2017 por la cláusula democrática del «Protocolo de Ushuaia». En agosto de 2017, 11 países latinoamericanos y Canadá firmaron la declaración de Lima que atacó el rompimiento de la orden democrática en Venezuela. Desde 2013, Venezuela renunció a la Convención Interamericana de Derechos Humanos.

La conclusión es que, por todas las violaciones a las libertades, al Estado democrático de derecho, problemas en la separación de poderes, ataques a la prensa y libertad periodísticas, violaciones sistemáticas de derechos humanos podemos considerar el régimen de chavista/ socialista autoritario.

\section{Factores que llevaron a esa situación}

el proceso de la caída de la democracia en Venezuela y la crisis humanitaria tiene una serie de razones para que pase lo que pasó. Es importante entender las ideas que crearon el chavismo y entendimiento del populismo y del socialismo (en la versión del siglo XXI). Entre los factores que llevaron a esa situación podemos hablar de los antecedentes del Pacto de Punto Fijo, la ascensión de Chávez, las medidas económicas neosocialistas y populistas, el apoyo político internacional de instituciones como el Foro de San Pablo, la caída de la libertad económica y civil, y la censura de la prensa libre.

Para empezar a entender la situación es importante tener los conceptos claves de populismo y de socialismo que iremos a trabajar. El concepto de populismo según Antonella Marty:

Estos gobiernos de carácter 'populista' tienen como característica principal a un mandatario que pretende convertirse en la figura central de la nación, presentándose como un mesías o una especie de salvador sensacional. Colocarse en el centro de la escena y contar con una buena cuota de carisma son características que jamás pueden escasear en una popularidad populista. El populista busca establecer en común para él y para la totalidad del pueblo. En otras palabras, el populista busca un chivo expiatorio a quien pueda culpar de todos los males que él mismo se ocupará de generar. Uno de los ejemplos más comunes de 'enemigo' en los populistas latinoamericanos es el famoso 'imperio yanqui, el capitalismo, el mercado, el 'neoliberalismo', los empresarios o la globalización (MARTY, 2018, p. 343)

Y el concepto de Socialismo según Huerta de Soto:

No plano material, o socialismo impede e dificulta em grande medida a produção de bens e serviços, constituindo-se, assim, como um obstáculo para o desenvolvimento econômico. Na área 
cultural, o socialismo tolhe a criatividade, impossibilitando o desenvolvimento e a aprendizagem de novos padrões de comportamento e dificultando a descoberta e a introdução de inovações. E, no campo científico, o socialismo não é senão um erro intelectual, que decorre da ideia de que a capacidade da mente humana é muito superior a que realmente tem e que, portanto, é possível obter informação precisa para melhorar a sociedade por meio de coerção. Em suma, o socialismo constitui a atividade anti-humana e antissocial por excelência, uma vez que se baseia na coerção sistemática contra a mais íntima e natural essência do ser humano: a sua própria capacidade para agir criativa e livremente. $(\underline{\mathrm{SOTO}, 2013, \text { p.99) }}$

Los conceptos de Soto de socialismo y de Marty nos ayudan a entender lo que fue el régimen chavista, no por sus ambiciones o promesas, sino por sus resultados y consecuencias. Es importante traer esos conceptos académicos basados en las experiencias concretas de los socialismos y no por las utopías que prometían llegar y el paraíso en la tierra. Ludwig von Mises ya había hablado de la imposibilidad del cálculo económico del socialismo y de cómo una autoridad central controlando cada vez más la distribución de la riqueza generarían más concentración de poder en la autoridad estatal y no libertad o democracia. Y es a partir de estas consideraciones que debemos analizar la experiencia chavista, y no como un paraíso utópico que salió mal, sino como una mala idea que genera malos resultados.

Con esos conceptos podemos llegar al momento político de la subida de Chávez. La Venezuela de 1998 era un país que venía de un período de larga experiencia democrática desde 1958 con el Pacto de Punto Fijo ${ }^{1}$ en cuanto casi todo el continente latinoamericano estaba en dictaduras. Sin embargo, la percepción de la población en Venezuela había empeorado mucho en la década de 1990 con la crisis del precio de petróleo y la incapacidad del sistema bipartidista de la Acción Democrática y la COPEI de contestar satisfactoriamente las demandas del pueblo venezolano. En ese contexto de deterioración económica, el teniente coronel Hugo Chavez Frias hizo un golpe militar fracasado en 1992 y fue a la cárcel por eso. Entretanto, ese intento de golpe militar hizo con que él ganase popularidad con el pueblo y también que quedase conocido por la gente. En 1994, Hugo Chávez fue libertado por el presidente Rafael Caldera y en ese mismo año fue recibido por el dictador cubano Fidel Castro como si fuera un jefe de Estado. Desde el intento de golpe, Fidel Castro ya percibía que Chávez sería una oportunidad para la isla cubana de obtener el petróleo venezolano (LLOSA; MACHADO, 2017).

Una institución internacional decisiva para el mantenimiento y la legitimación internacional de Hugo Chávez y Nicolas Maduro en el poder fue el Foro de San Pablo. La organización fue creada en 1990 por Fidel Castro y Lula da Silva, su proyecto pretendía fortalecer los partidos socialistas, antiimperialistas y revolucionarios de la región. Buscaba permitir el dialogo y apoyo intercontinental entre esos partidos. La interferencia del gobierno cubano en Venezuela no fue apenas por el Foro de San Pablo sino también con los millares de agentes cubanos en territorio venezolano y vinculado directamente con Miraflores. El escritor y PhD en Filosofía Enrique Serrano (2018) explica el nacimiento y algunas acciones del Foro de San Pablo, incluso los cambios institucionales y constitucionales que tantas veces serian hechos por el Socialismo de Siglo XXI:

\footnotetext{
${ }^{1}$ Con todos los problemas que había en la sociedad venezolana, el sistema democrático mantuvo funcionando como una democracia más o menos estable.
} 
Una de las herramientas recurrentes y fundamentales del Foro de San Pablo estriba en encomendar a todos los actores y partidos de izquierda que lleguen al poder, es la convocatoria inmediata y fulminante de asambleas constituyentes que les permitan crear un marco legal favorable para la izquierda radical, hecho a la medida de sus propósitos políticos: producir la impresión de una nación nueva, «rebelde e independiente», dotada de un marco normativo que niegue - o limite seriamente - las libertades económicas y el libre crecimiento de economías de mercado integradas al sistema global. La estatización constitucional y el uso incontrolado del gasto público son las reglas en tales empeños refundadores. (LOPEZ; PARDO, 2018, p. 210)

Además de la interprotección entre los partidos y países que integraban el Foro de San Pablo, hubo financiamiento recíproco con uso de bancos gubernamentales para objetivos partidarios que fortalecían el movimiento continental. Como en las muchas obras de Odebrecht con financiamiento del BNDES en Brasil y apoyo político del PT (Partido de los Trabajadores, en Brasil); o con los financiamientos con los petrodólares de Chávez en toda la América Latina.

En términos constitucionales y económicos, el Chavismo fue progresivamente destruyendo cualquier resquicio de economía de mercado, y actuando con el Estado fuertemente en la economía con leyes como la ley del precio máximo (ABADI, 2018), que diferentemente de su intención lo que logró fue hacer con que Venezuela tuviera la inflación más grande del mundo. El control, el aumento gigantesco del número de funcionarios y la estatización de la PDVSA fue otro elemento fundamental para causar la gran crisis económica que hoy existe en Venezuela. Los incentivos para la producción de empresas estatales ya son naturalmente peores para la producción de sus bienes; pero en el socialismo del Siglo XXI, PDVSA fue usada para corrupción, clientelismo político interno e internacionalmente (DIARIO DE LAS AMERICAS, 2018). Otra causa de los problemas económicos de Venezuela es la caída substancial en la libertad económica - ( HERITAGE FOUNDATION, 2018) Venezuela está en la posición 179 de 180 países, quedando delante apenas de Corea del Norte- y está en pésima posición en facilidades para hacer negocios (DOING BUSINESS REPORT, 2018), está en la posición 188 de 190 países, atrás de países en guerra como Irak, Libia, Congo y otros.

$\mathrm{Y}$ por fin, debemos citar que las políticas de expropiaciones fueron decisivas para el caos económico, social y humanitario que vive el país latinoamericano. Las políticas de expropiaciones acaban con los incentivos a las inversiones y estimula la salida de empresas del país. Además de eso, los incentivos económicos para las empresas estatales son mucho más políticos, generan gran ineficiencia y corrupción.

\section{Referencias}

ABADI, Anabella M., C. G. Prodavinci. 2018. Obtenido en: https://prodavinci.com/15-anos-del-actual-controlde-precios-en-venezuela-un-balance/. Acceso en: 4 mar 2019.

DAHL, R. La poliarquia: participación y oposición. Tecnos. Online edition. 1989. Obtenido en: https://edisciplinas. usp.br/pluginfile.php/4229936/mod_resource/content/1/RobertDahl_Poliarquia_espanhol.pdf. Acceso en: 4 mar 2019.

DIARIO DE LAS AMERICAS. Investigación revela la opulencia del chavismo. Diario de las Americas. 2018. Obtenido en: https://www.diariolasamericas.com/america-latina/investigacion-revela-la-opulencia-del-chavismoespana-n4162027. Acceso en: 4 mar 2019 
DOING BUSINESS REPORT. Doing Business. 2018. Obtenido en: http://www.doingbusiness.org/content/dam/ doingBusiness/media/Annual-Reports/English/DB2018-Full-Report.pdf. Acceso en: 4 mar 2019

FRASIER INSTITUTE. Economic Freedom of the World. 2017. Obtenido en: https://www.fraserinstitute.org/ sites/default/files/economic-freedom-of-the-world-2017.pdf. Acceso en: 4 mar 2019

FRASIER INSTITUTE. Human Freedom Index. 2017. Obtenido ene: https://www.fraserinstitute.org/sites/default/ files/human-freedom-index-2017-web.pdf. Acceso en: 4 mar 2019.

HERITAGE FOUNDATION. Countries: Venezuela. Heritage Foundation. 2018. Obtenido de: https://www. heritage.org/index/pdf/2018/countries/venezuela.pdf. Acceso en: 4 mar 2019.

HUMANS RIGHT WATCH WORLD REPORT. Humans Right Watch World Report. 2018. Obtenido en: https:// www.hrw.org/es/world-report/2018/country-chapters/313312. Acceso en: 4 mar 2019.

THE ECONOMIST. Indice de Democracia. The Economist. 2017. Obtenido en: https://pages.eiu.com/rs/753RIQ-438/images/Democracy_Index_2017.pdf. Acceso en: 4 mar 2019

LEVITSKY, S. Elections Without Democracy. The Rise of Competitive Authoritarianism. Journal of democracy, v.13. n.2, 2002. Acceso en: 4 mar 2019.

LLOSA, A. V. ; MACHADO, M. C. El estallido del populismo. Bogotá: Planeta, 2017.

LOPEZ, E. S.; PARDO, C. N. Socialismo latino americano: notas críticas. Bogotá: DGP Editores, 2018.

MARTY, A. Lo que todo revolucionario del siglo XXI tiene que saber. Bogotá: Unión Editorial Colombia, 2018. REPORTEROS SIN FRONTERAS. Classificación Mundial. Disponible en: https://www.rsf-es.org/grandes-citas/ clasificacion-por-paises/. Acceso en: 4 mar 2019.

RULE OF LAW INDEX. World Justice Project. 2018. Obtenido en: https://worldjusticeproject.org/sites/default/ files/documents/WJP-ROLI-2018-June- ADDIN ZOTERO_BIBL \{“uncited":[],"omitted":[],"custom":[]\} CSL_ BIBLIOGRAPHY. Acceso en: 4 mar 2019.

SOTO, J. H. Socialismo, Cálculo econômico e função empresarial. São Paulo: Instituto Ludwig Von Mises Brasil, 2013.

RECEBIDO EM: 23/10/2018

APROVADO EM: 03/12/2018 